\title{
Authorized Investigational Medicinal Product
}

National Cancer Institute

\section{Source}

National Cancer Institute. Authorized Investigational Medicinal Product. NCI Thesaurus.

Code C156617.

A medicinal product with marketing authorization that is being tested or used as a

reference in a clinical trial. 